\title{
Temperature stability of elastic and piezoelectric properties in $\mathrm{LiNbO}_{3}$ single crystal
}

\author{
I Franke ${ }^{1}$, E Talik ${ }^{1}$, K Roleder ${ }^{1}$, K Polgár ${ }^{2}$, J P Salvestrini ${ }^{3}$ and \\ M D Fontana ${ }^{3}$ \\ ${ }^{1}$ Institute of Physics, University of Silesia, ul. Uniwersytecka 4, 40-007 Katowice, Poland \\ ${ }^{2}$ Research Institute for Solid State Physics and Optics, Hungarian Academy of Sciences, \\ Konkoly Thege ut 29-33, 1121 Budapest, Hungary \\ ${ }^{3}$ LMOPS, UMR CNRS 7132, University of Metz and Supelec, 2 rue E. Belin, 57070 Metz, \\ France
}

\begin{abstract}
Temperature dependences of the piezoelectric modulus $d_{31}$ and complex elastic compliance $s_{11}^{\mathrm{E}}$ have been measured for a lithium niobate single crystal grown by the high temperature top-seeded solution growth technique. The results obtained have shown that the imaginary part of the elastic coefficient is probably sensitive to the lithium ion and proton migration inside the crystal. X-ray photoelectron spectroscopy has revealed the existence of some defects in the crystal structure. While these imperfections only weakly control the magnitude of the piezoelectric coefficients, they influence the elastic properties, even in the relatively low temperature range. It was found that the temperature dependence of the elastic properties is stable as long as a temperature of $\sim 90^{\circ} \mathrm{C}$ is not reached.
\end{abstract}

\section{Introduction}

Lithium niobate $\mathrm{LiNbO}_{3}(\mathrm{LN})$ is one of the most widely used materials in electro-optics and in optical devices. In this field of applications the presence of defects in the crystal lattice can significantly modify the optical properties [1,2]. $\mathrm{LN}$ is a typical non-stoichiometric material with the ratio $\mathrm{Li} /(\mathrm{Li}+\mathrm{Nb})<50 \%$ and this causes a high concentration of intrinsic defects. The possible defects which satisfy charge compensation have been discussed in the literature: the formation of $\mathrm{Li}$ and $\mathrm{O}$ vacancies [4], excess of $\mathrm{Nb}^{5+}$ ions substituted for $\mathrm{Li}^{+}$sites ( $\mathrm{Li}$ vacancies are then produced) [5] and $\mathrm{Nb}$ vacancies [6]. According to Feng et al [7], in nearly stoichiometric LN, Li Frenkel defects mainly exist while Li vacancies dominate in non-stoichiometric material.

LN single crystals can be grown from congruent melt by the Czochralski method but their composition is not stoichiometric [5]. The humidity of the surrounding atmosphere during crystal growth may cause the incorporation of the $\mathrm{OH}^{-}$group [9]. On the other hand, some traces of $\mathrm{OH}^{-}$in the crystal may originate from the raw materials, probably from the $\mathrm{Li}_{2} \mathrm{O}$. Since $\mathrm{OH}^{-}$ions have nearly the same radius as $\mathrm{O}^{2-}$, it is possible for both of them to occupy $\mathrm{O}^{2-}$ and interstitial sites. In the former case it represents positive charge with respect to the unperturbed lattice, as a result of the valence change from $\mathrm{Nb}^{5+}$ to $\mathrm{Nb}^{4+}$ or $\mathrm{Li}$ vacancies are created [9]. In recent years, a few growth techniques have been developed enabling the growing of LN crystals with compositions close to the stoichiometric one and having good quality (e.g. [3, 8, 10-13]). The high temperature top-seeded solution growth (HTTSSG) technique is one of them.

Because of all the factors mentioned above, there are chemical composition fluctuations in LN crystals. The piezoelectric effect, which plays a role in the electro-optical properties, can also be influenced by the defects appearing in the lattice. Through the observation of temperature changes of the piezoelectric resonances, the influence of defects on elastic properties can be observed. To check crystal homogeneity and to identify the type of defects, XPS spectroscopy has been applied.

\section{Experimental conditions}

Experiments have been conducted on an LN crystal prepared by the HTTSSG technique [3], which enables single-domain 

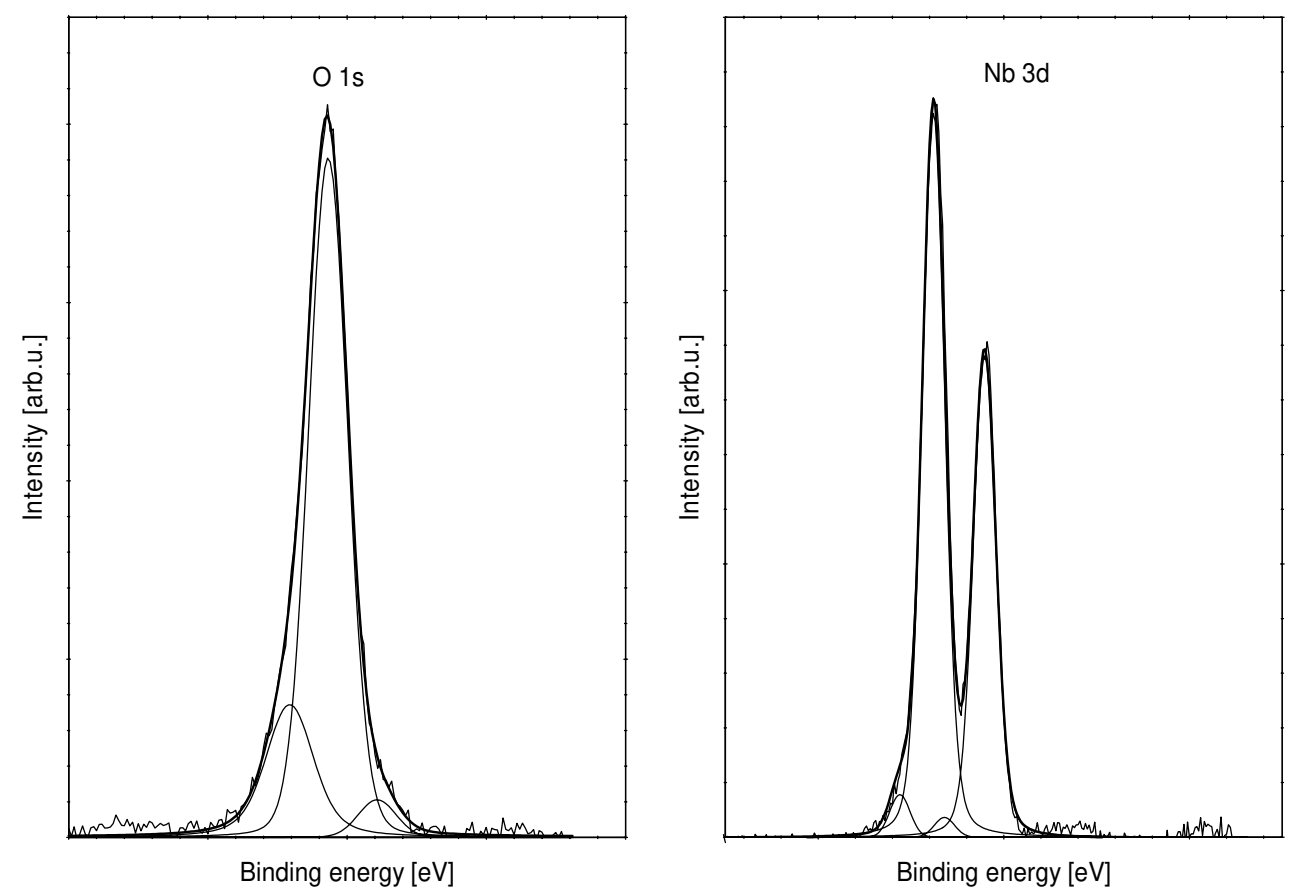

Figure 1. Comparison of the $\mathrm{O} 1 \mathrm{~s}$ and $\mathrm{Nb} 3 \mathrm{~d}$ states registered for the crystal grown by the HTTSSG technique.

crystals to be obtained without additional poling [8]. Bar-shaped samples having a geometry appropriate for piezoelectric measurement [14] were cut from the single crystal. Gold electrodes were evaporated on the surfaces perpendicular to the spontaneous polarization direction [001]. Electrical contact was accomplished via thin silver wires glued to the electrodes.

The XPS spectra were recorded over a wide energy range at different places on the $\mathrm{LN}$ single crystal using a PHI 5700/660 physical electronics photoelectron spectrometer with monochromatized $\mathrm{Al} \mathrm{K}_{\alpha}$ x-ray radiation $(1486.6 \mathrm{eV})$. All the measurements were performed under UHV conditions $\left(10^{-10}\right.$ Torr $)$ and with a neutralizer discharging non-conducting samples. The binding energy was determined with reference to the $\mathrm{C} 1 \mathrm{~s}$ line at $284.8 \mathrm{eV}$ for the broken crystal. The Multipak Physical Electronics programme allowed the quantification of the XPS spectra using peak area and peak height sensitivity factor. The peaks were deconvoluted after background subtraction by applying a Lorenzian function with no asymmetry. Element concentrations with the accuracy limit better than $10 \%$ were calculated by the standard atomic method, i.e. as the ratio of the parameter for each component line to the sum of those for all elemental lines visible in the spectrum.

The piezoelectric resonance method allowed the determination of the real and imaginary parts of elastic compliance $s_{11}^{\mathrm{E}}$, piezoelectric modulus $d_{31}$ and electromechanical coupling factor $k_{31}$. To calculate these coefficients the forced harmonic oscillator model was applied [15]. The piezoelectric resonances have been found by measurement of the complex admittance $Y=G+\mathrm{i} B$ as a function of frequency $f=\omega / 2 \pi$ by means of a Hewlett-Packard 4192A impedance analyser.

The procedure for the measurements was as follows. Several samples of LN crystal grown by the HTTSSG technique have been used to test the temperature stability of the piezoelectric resonances. It was recognized that above $100^{\circ} \mathrm{C}$ the single piezoelectric resonance has been transformed into a multi-resonance one. Due to this, for a sample with no thermal history first a sequence of the thermal cycles (on heating and cooling) has been performed in the range $25-100^{\circ} \mathrm{C}$. After the first cycle a time evolution of piezoelectric resonance in $90 \mathrm{~min}$ has also been observed at ambient temperature. After four thermal cycles the temperature changes of the piezoelectric resonance have in turn been measured on heating (up to $180^{\circ} \mathrm{C}$ ) and next on cooling to ambient temperature.

\section{X-ray photoelectron spectroscopy}

The composition of the crystal studied agrees with the nominal formula within the accuracy limit of the standard atomic method. The position of the $\mathrm{Nb} 4 \mathrm{~s}$ line $(56 \mathrm{eV})$ is very close to the $\mathrm{Li} 1 \mathrm{~s}$ one $(54.8 \mathrm{eV})$, and that is why this method is not able to establish with satisfactory accuracy the ratio Li/ $\mathrm{Nb}$. According to Polgar et al [8] for crystals grown by the HTTSSG technique, this ratio is very close to one and it is one of the reasons for better structure appearing in this crystal (figure 1). The narrow but asymmetric $\mathrm{O} 1 \mathrm{~s}$ line (figure 1(a)) may be decomposed into three lines: one corresponds to oxygen bonding in the proper structure and two additional lines confirm the existence of the oxygen defects or some contaminations [16]. The shape of the $\mathrm{Nb} 3 \mathrm{~d}$ line (figure 1(b)) is a superposition of two doublets: the stronger one is due to the $\mathrm{Nb}^{5+}$ state and the second, at lower binding energy, due to the $\mathrm{Nb}^{4+}$ state $[17,18]$. These kinds of defects have been found at different places on the LN single crystal grown by the HTTSSG technique. Thus it is interesting if the defective crystal lattice as described above can influence the elastic and piezoelectric 


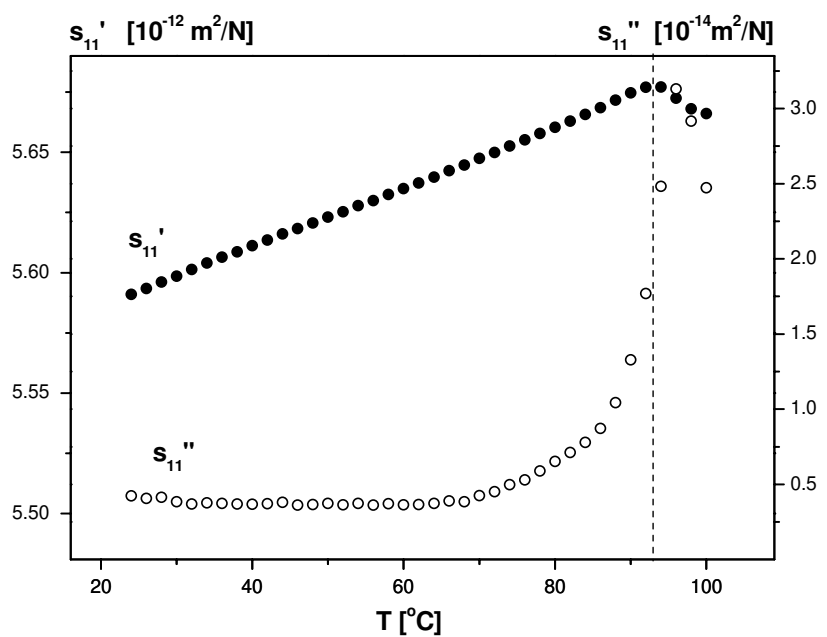

Figure 2. Temperature dependences of the real $s_{11}^{\prime}$ and imaginary $s_{11}^{\prime \prime}$ parts of the elastic compliance obtained for crystal grown by HTTSSG technique in the first cycle on heating.

properties, contributing strongly to the electro-optical activity of the LN [2].

\section{Elastic and piezoelectric properties}

The temperature behaviour of the complex elastic compliance $s_{11}^{\mathrm{E}}$ (figure 2) shows that the real part increases smoothly revealing an anomaly at $\sim 90^{\circ} \mathrm{C}$. The maximum on the temperature evolution of the imaginary part lies slightly above $s_{11}^{\prime}(T)$ local maximum. This peak in $s_{11}^{\prime \prime}(T)$ may be related to the absorption of elastic energy due to the defects. It was observed that LN single crystals of disordered structure had maxima in $s_{11}^{\prime \prime}(T)$ and $s_{11}^{\prime}(T)$ at lower temperature [19]. Several reasons could be invoked to explain these maxima. For example, crystals obtained by the Czochralski method contained more Li vacancies [7], and thus more $\mathrm{OH}^{-}$ions (or protons) are incorporated into the lattice [9].

According to Klauer et al [20] the migration of the protons influences the temperature stability of holograms via the dark conductivity. Mandula et al [21] observed the time changes of the shape of the $\mathrm{OH}^{-}$band in $\mathrm{LN}$ until the moving protons reach a thermodynamic equilibrium at a given temperature. Protons migrate among the different sites but the total proton concentration does not change [21]. Thus the time evolution of piezoelectric resonance at $25^{\circ} \mathrm{C}$ observed by us after the first cooling from $100^{\circ} \mathrm{C}$ was probably a proof for this proton migration because the signal was becoming sharper and reached a final shape after $90 \mathrm{~min}$. Hence, after heating the crystal the thermodynamic equilibrium was destroyed resulting in characteristic maxima in $s_{11}^{\prime \prime}(T)$ [19]. By an infrared absorption study of the $\mathrm{OH}^{-}$absorption bands Kovacs et al [1] found that the temperature of about $100^{\circ} \mathrm{C}$ was distinguished in $\mathrm{LN}$ crystals.

Heating this crystal four times to $100{ }^{\circ} \mathrm{C}$ caused further anomalies to appear in $s_{11}^{\prime}(T)$ and $s_{11}^{\prime \prime}(T)$ (figure 3 ). Moreover, in the next thermal cycle the $s_{11}^{\prime \prime}(T)$ run became similar to those observed for the crystals having the disordered structure [19]. As an example, data presented for the first and fourth cycles in figure 3 show that the $s_{11}^{\prime}(T)$ and $s_{11}^{\prime \prime}(T)$ anomalies originally
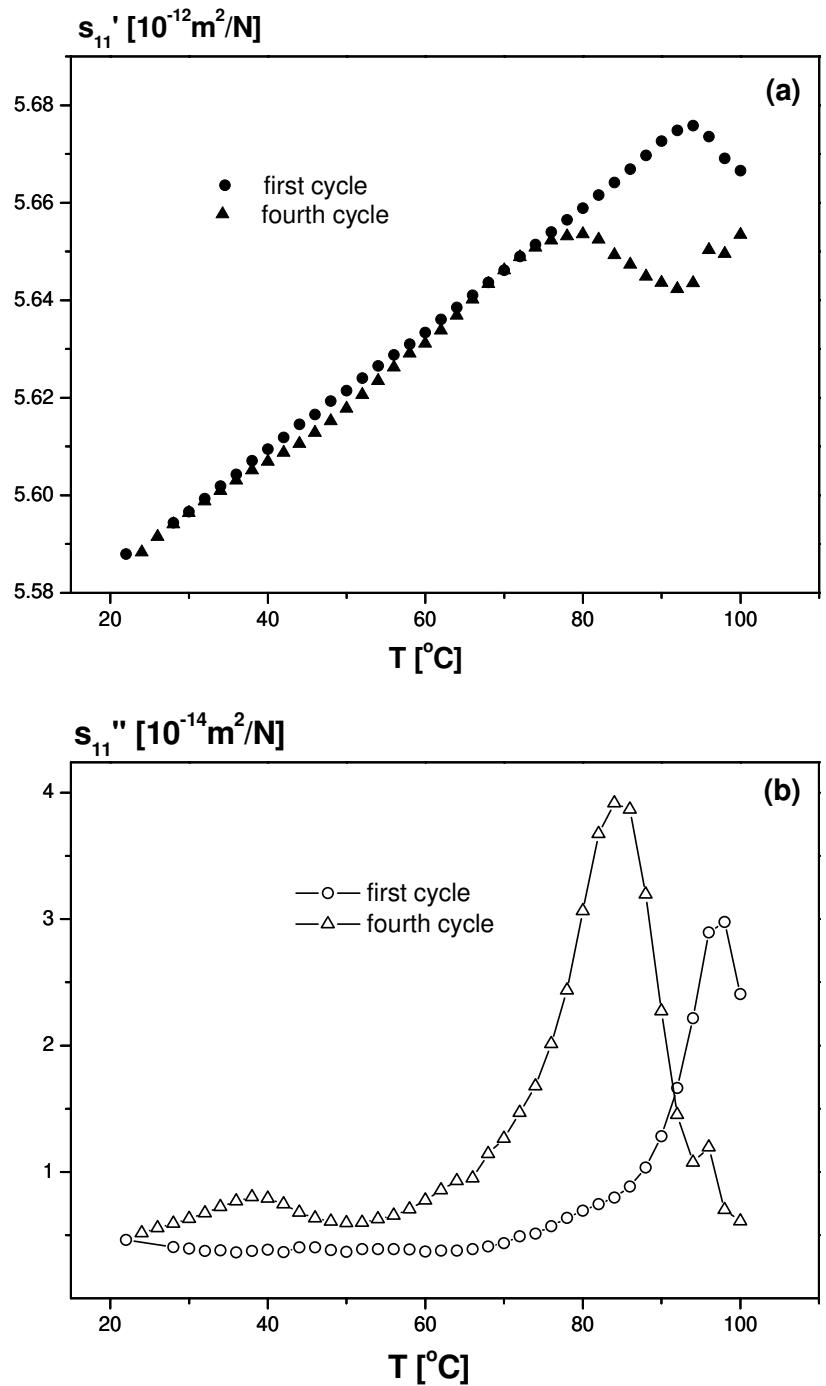

Figure 3. Temperature dependence of the real $s_{11}^{\prime}(a)$ and imaginary $s_{11}^{\prime \prime}(b)$ parts of the elastic compliance obtained for a crystal grown by the HTTSSG technique in two different thermal cycles on heating.

placed at $\sim 90^{\circ} \mathrm{C}$ shifted down by about $15^{\circ}$. Moreover a new maximum in $s_{11}^{\prime \prime}(T)$ appeared at $\sim 40^{\circ} \mathrm{C}$ (figure $3(b)$ ).

It is very probable that above $\sim 90^{\circ} \mathrm{C}$, on account of the high temperature, intensive proton migration induces Li ion motion and as a consequence the change of the polarization state. The impurity segregation and developing mechanical stress may promote the reversal of the domain pattern in the crystal [3]. This is probably the reason that the additional piezoelectric resonances appeared for the crystal heated up to $180^{\circ} \mathrm{C}$. An interchange between the intensities of the resonances $\mathrm{A}$ and $\mathrm{B}$ related with the piezoelectric $d_{31}$ mode is clearly seen in figure 4 . At $120^{\circ} \mathrm{C}$ the A resonance is smaller than $\mathrm{B}$ and finally at ambient temperature the $\mathrm{A}$ resonance dominates. Because of the splitting of the resonance it was not reasonable to calculate the piezoelectric coefficients.

It is worth mentioning that shifts of the $s_{11}^{\prime}(T)$ and $s_{11}^{\prime \prime}(T)$ were obtained for a $\mathrm{Li}_{2} \mathrm{~B}_{4} \mathrm{O}_{7}$ (LTB) single crystal. However, vice versa as for LN crystal, in succeeding thermal cycles the anomalies of the $s_{11}^{\prime \prime}(T)$ runs have been shifted to higher temperatures [22]. Although the Li ions enter into the 


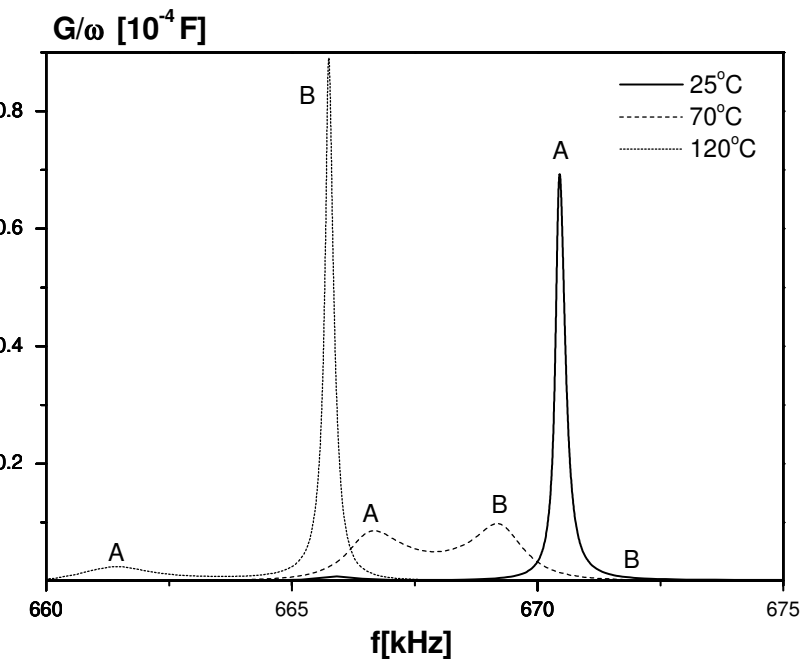

Figure 4. Temperature evolution of piezoelectric signal registered on cooling in the $d_{31}$ configuration for crystal heated up to $180^{\circ} \mathrm{C}$. $\mathrm{A}$ and $\mathrm{B}$ indicate two different resonances.

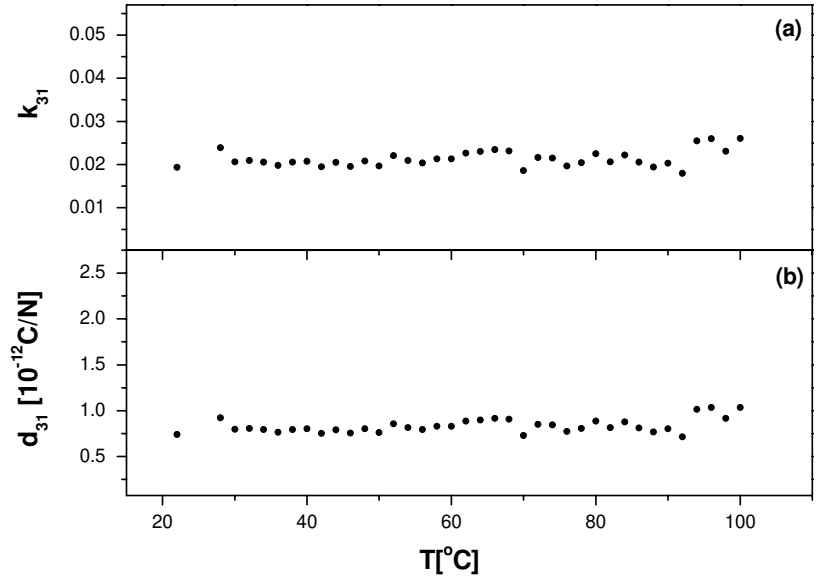

Figure 5. (a) Temperature behaviour of electromechanical coupling factor, $k_{31}$, and $(b)$ piezoelectric modulus, $d_{31}$, for the crystal obtained by the HTTSSG technique. The values of $d_{31}$ and $k_{31}$ have been calculated in the first thermal cycle on heating (see figure 3 ).

composition of these crystals their role in the lattice dynamics because of the different structures of the LN and LTB may be different.

The main results from the experiments are connected with the fact that for this crystal the temperature evolution of the real and imaginary parts of the elastic compliance $s_{11}^{\mathrm{E}}$ was repeatable in thermal cycles up to $\sim 90^{\circ} \mathrm{C}$ only.

Though temperature instability of elastic properties in the LN crystals may appear, the values of piezoelectric coefficient $d_{31}$ and $k_{31}$ (figure 5) are almost independent of temperature and number of thermal cycles if the sample is not heated far above $100^{\circ} \mathrm{C}$. It would confirm that the concentration of defects created during the technological process weakly changes the values of these coefficients and is low enough not to change their temperature stability.

The piezoelectric effect plays an essential role in the electro-optical effect of ferroelectric materials. This electrooptic coefficient is considered as a sum of three components, the contribution of which depends on the frequency of the modulating electric field in relation to the frequency of the acoustic (piezoelectric) resonance, lattice phonons and electrons. In some applications, the modulating field is of low frequency, lower than the acoustic resonances, and thus the piezoelectric effect is determined by the magnitude of the so-called secondary electro-optical (piezo-optic) effect. In inorganic ferroelectric materials the secondary effect due to the photoelasticity and the piezoelectricity generally preponderates over the electronic and lattice contributions. Hence, the piezoelectric tensor is indispensable to evaluate the electro-optical effect for low-frequency electric fields. On the basis of experiments described in this paper, i.e. the temperature stability of elastic and piezoelectric properties, it would mean that the contribution of the piezo-optic effect in an LN single crystal should be stable and temperature independent provided the crystal is not heated above $\sim 90^{\circ} \mathrm{C}$.

\section{Conclusions}

The influence of defects on the elastic and piezoelectric properties of the LN crystal prepared by the HTTSSG technique has been studied. Investigations have shown the appearance of anomalies on the temperature dependences of the real and the imaginary parts of the elastic tensor which shift towards lower temperatures in subsequent thermal cycles. This thermal instability occurs because of the presence of chemical and structural imperfections in the lattice. For the crystal investigated it was found that the elastic characteristics are reproducible provided the temperature does not exceed about $90{ }^{\circ} \mathrm{C}$. On the other hand, the piezoelectric response does not depend on the temperature and the number of thermal cycles, provided the crystal is not heated far above $100^{\circ} \mathrm{C}$. Thus we can conclude that the measured piezoelectric coefficients are weakly susceptible to the defect concentration in this temperature range. It is important from the point of view of the piezo-optic effect that may strongly contribute to the value of the electro-optic coefficient in the LN single crystals.

\section{Acknowledgment}

The paper was co-financed by the State Committee for Scientific Research (KBN) under grant 2 P03B 06425 in the years 2003-2006.

\section{References}

[1] Kovács L, Wöhlecke M, Jovanovicv, Polgárv K and Kapphan S 1991 J. Phys. Chem. Solids 52797

[2] Chah K, Fontana M D, Aillerie M, Bourson P and Malovichko G 1998 Appl. Phys. B 6765

[3] Polgár K, Peter A and Földvári I 2002 Opt. Mater. 197

[4] Fay H, Alford W J and Dess H M 1968 Appl. Phys. Lett. 1289

[5] Lerner P, Legras C and Dumas J P 1968 J. Cryst. Growth 3/4 231

[6] Abrahams S C and Marsh P 1986 Acta Cryst. B 4261

[7] Feng S, Jin Q, Li B, Guo Z and Ding D 2000 Preprint cond-mat/0012499 v1

[8] Polgár K, Peter A, Kovács L, Corradi G and Szaller Z 1997 J. Cryst. Growth 177211

[9] Bollmann W and Stohr H J 1977 Phys. Status Solidi a 39477 
[10] Jeon O Y, Kim H K, Kim T H, Ro J H and Cha M 2001 J. Korean Phys. Soc. 38138

[11] Kitamura K, Yamamoto J K, Iyi N, Kimura S and Hayashi T 1992 J. Cryst. Growth 116327

[12] Bordui P F, Norwood R G, Jundt D H and Fejer M M 1992 J. Appl. Phys. 71875

[13] Luh Y S, Fejer M M, Byer R L and Feigelson R S 1987 J. Cryst. Growth $\mathbf{8 5} 264$

[14] Bauer A, Buhling D, Gesemann H J, Helke G and Schreckenbach W 1976 Technology and Application of Ferroelectrics (Leipzig: Akademische Verlagsgesellschaft) p 322

[15] Roleder K, Franke I, Glazer A M, Thomas P A, Miga S and Suchanicz J 2002 J. Phys.: Condens. Matter 145399
[16] Talik E, Kruczek M, Sakowska H, Ujma Z, Gała M and Neumann M 2004 J. Alloys Compounds 377259

[17] Szot K, Keppels J, Speier W, Besocke K, Teske M and Eberhardt W 1993 Surf. Sci. 280179

[18] Szot K, Hillebrecht F U, Sarma D D, Campagna M and Arend H 1986 Appl. Phys. Lett. 48490

[19] Franke I, Salvestrini J P, Fontana M and Roleder K 2004 Ferroelectrics 304155

[20] Klauer S, Wöhlecke M and Kapphan S 1992 Phys. Rev. B 452786

[21] Mandula G, Lengyel K, Kovács L, Ellabban M A, Rupp R A and Fally M 2001 Proc. SPIE 4412226

[22] Franke I, Roleder K, Miga S and Maillard A 2002 Phase Transit. $\mathbf{7 5} 631$ 